\title{
Token-Based Authenticated Key Establishment Protocols for Three-Party Communication
}

\author{
Eun-Jun Yoon ${ }^{1}$ and Kee-Young Yoo ${ }^{2, \star}$ \\ ${ }^{1}$ Faculty of Computer Information, Daegu Polytechnic College, \\ 42 Jinri-2gil (Manchon 3dong San395), Suseong-Gu, Daegu 706-711, South Korea \\ ejyoon@tpic.ac.kr \\ 2 Department of Computer Engineering, Kyungpook National University, \\ 1370 Sankyuk-Dong, Buk-Gu, Daegu 702-701, South Korea \\ Tel.: +82-53-950-5553; Fax: +82-53-957-4846 \\ yook@knu.ac.kr
}

\begin{abstract}
This paper proposes new efficient and secure three-party token-based authenticated key establishment (3TAKE) protocols based on symmetric key cryptosystem to minimize the complexity of symmetric key encryption/decryption operation among all users and fit three-party communication. In 3TAKE, the number of exponentiations among three parties is same or reduced by about $34 \sim 60 \%$ and the number of symmetric key encryption/decryption operations among three parties is reduced by about $34 \sim 67 \%$ compared with the related protocols, respectively. Furthermore, the number of rounds is one round smaller than the related protocols and the asymmetric key encryption/decryption operations do not need to establish a session key and authenticate between two users and a server.
\end{abstract}

Keywords: Cryptography, Token, Authentication, Password, Key agreement, Three-party.

\section{Introduction}

\subsection{User Authentication}

User authentication is a process that verifies a user's identity to ensure that the person requesting access to the private network is in fact, that person to whom entry is authorized. Generally, there exist three kinds of approaches for user authentication [1|3/45]:

1. Password-based user authentication ("what you know"): Passwords and PINs are examples of this approach.

2. Token-based user authentication ("what you have"): This approach includes physical keys, ATM or smart cards, tokens, mobile devices (cell phones, PDA, sensor nodes) and so on.

3. Biometric user authentication ("what you are"): Voice, fingerprints, retinal scans, and keystrokes are included in this approach.

\footnotetext{
^ Corresponding author.
} 


\section{$1.2 \quad$ Authenticated Key Establishment}

Authenticated key establishment (AKE) protocols are designed to provide two or more specified entities communicating over an open network with a shared secret key which may subsequently be used to achieve some cryptographic goal such as confidentiality or data integrity [6. Key establishment may be broadly subdivided into key transport and key agreement that may be employed to establish session keys [2].

- Key transport protocol: A key transport protocol is a key establishment technique where one party creates or otherwise obtains a secret value, and securely transfers it to the other(s).

- Key agreement protocol: A key agreement protocol is a key establishment technique in which a shared secret is derived by two (or more) parties as a function of information contributed by, or associated with, each of these, (ideally) such that no party can predetermine the resulting value.

\subsection{Three-Party Communication Environment}

In a three-party (a server and two clients) setting, key transport protocol means that the session key is created by the server and securely transmitted to these two clients, and key agreement protocol means that both clients contribute information to derive the common session key.

Recently some researches proposed three-party AKE protocols. However, these protocols are insecure some attacks and inefficiently designed because of preforming many symmetric key encryption/decryption operations. Gong, Lomas, Needham, and Saltzer [7 proposed a protocol, called GLNS protocol, in a three-party setting in which two users establish a session key through an authentication server. Timestamps are used in the protocol to guarantee message freshness. By using nonces and confounders, the protocol is successful in generating a large search space to resist off-line password guessing attacks. Many protocols 8 [9]10[11]12]13]16] have been addressed to discuss this problem.

\subsection{Our Contributions}

This paper proposes new efficient and secure three-party token-based authenticated key establishment (3TAKE) protocol based on symmetric key cryptosystem to minimize the complexity of symmetric key encryption/decryption operation among all users and fit three-party communication. The proposed 3TAKE protocols have several important features as follows:

- The proposed 3TAKE protocols are designed to reduce the computation cost of each participant by using the small number of exponentiations.

- The proposed 3TAKE protocols achieve cryptographic goals only using symmetric key cryptosystems, exponentiations and collision-free one-way hash functions as main cryptographic operations without additional requirements such as using server's public key, digital signatures, and so on. 
- The proposed 3TAKE protocols use the bitwise exclusive-OR operation and symmetric key cryptosystems to authenticate each other among parties. Additionally, this allows the protocols simply prevent off-line password guessing attack.

- The proposed 3TAKE protocols not only are secure against well-known cryptographical attacks but also provide perfect forward secrecy.

In $3 \mathrm{TAKE}$, the number of exponentiations among three parties is same or reduced by about $34 \sim 60 \%$ and the number of symmetric key encryption/decryption operations among three parties is reduced by about $34 \sim 67 \%$ compared with the related protocols, respectively. Furthermore, the number of rounds is smaller one round than the related protocols and the asymmetric key encryption/decryption operations do not need to establish a session key and authenticate between two users and a server.

The remainder of this paper is organized as follows: In the next section, we provide preliminary information of 3TAKE. The proposed 3TAKE protocol is presented in Section 3, while Sections 4 and 5 discuss the security and efficiency of the proposed 3TAKE protocol. The Conclusion is given in Section 6 .

\section{Preliminary Information}

This section summarizes the underlying primitives used throughout this paper.

\subsection{Notations}

Some of the notations used in the proposed 3TAKE are defined as follows:

- $A, B, S$ : Two users and remote server, respectively.

- $I D_{A}, p w_{A}$ : User $A$ 's identifier and password, respectively.

$-I D_{B}, p w_{B}$ : User $B$ 's identifier and password, respectively.

$-x: S$ 's strong secret key.

$-r_{i}$ : Fresh random nonce.

- $p$ : Large prime (usually at least 1024 or 2048 bits).

- $q$ : Relatively small prime (typically of 160 bits) with $q \mid p-1$.

$-Z_{q}$ : Subgroup of order $q$ of $Z_{p}^{*}$.

- $g$ : Generator $g$ of $Z_{q}$.

- $a, b$ : Session-independent random exponents $\in[1, q-1]$ chosen by $A$ and $B$, respectively.

- $s k$ : Shared fresh session key computed by $A$ and $B$.

- $E_{v_{i}}(M)$ : Symmetric key encryption of message $M$ by using secret key $v_{i}$.

- $h(\cdot), f(\cdot)$ : Collision resistant secure one-way hash function with an output size of 512 bits, e.g. SHA-512.

- $\oplus$ : Bit-wise exclusive-OR (XOR) operation.

\subsection{Review of Related Protocols}

This subsection briefly reviews the related three-party AKE protocols 11129 and then point out the efficiency problems of their protocols. 
(1) LSH's three-party key agreement protocol [11]: The following protocol shows LSH's three-party password-based authenticated key agreement protocol. LSH's protocol requires totally 2 times asymmetric encryption and 2 times asymmetric decryption.

- $K_{S}: S$ 's public key.

1. $A \rightarrow B: I D_{A},\left\{r_{A}, g^{a}, p w_{A}\right\}_{K_{S}}$

2. $B \rightarrow S: I D_{A},\left\{r_{A}, g^{a}, p w_{A}\right\}_{K_{S}},\left\{r_{B}, g^{b}, p w_{B}\right\}_{K_{S}}$

3. $S \rightarrow B: E_{r_{A}}\left(B, g^{b}\right), E_{r_{B}}\left(A, g^{a}\right)$

4. $B \rightarrow A: E_{r_{A}}\left(B, g^{b}\right), E_{s k}\left(h(\right.$ flow 1$)$, Nonce $\left._{B}\right)$

5. $A \rightarrow B:$ Nonce $_{B}$

(2) LSSH's three-party key agreement protocol [12]: The following protocol shows LSSH's three-party password-based authenticated key agreement protocol. LSSH's protocol requires totally 7 rounds and six exponentiation operations.

- $S_{1}, S_{2}$ : Session independent random exponents chosen by $S$.

- $K_{A, S}$ : Shared session key computed by $A$ and $S$.

- $K_{B, S}$ : Shared session key computed by $B$ and $S$.

1. $A \rightarrow S: I D_{A}, I D_{B}$

2. $S \rightarrow A: E_{p w_{A}}\left(g^{S_{1}}\right), E_{p w_{B}}\left(g^{S_{2}}\right)$

3. $A \rightarrow B: I D_{A}, g^{a}, f_{K_{A, S}}\left(I D_{A}, I D_{B}, g^{S_{1}}\right), E_{p w_{B}}\left(g^{S_{2}}\right)$

4. $B \rightarrow S: g^{a}, f_{K_{A, S}}\left(I D_{A}, I D_{B}, g^{S_{1}}\right), g^{b}, f_{K_{B, S}}\left(I D_{A}, I D_{B}, g^{S_{2}}\right)$

5. $S \rightarrow B: f_{K_{B, S}}\left(I D_{A}, I D_{B}, g^{a}, g^{b}\right), f_{K_{A, S}}\left(I D_{A}, I D_{B}, g^{b}, g^{a}\right)$

6. $B \rightarrow A: g^{b}, f_{K_{A, S}}\left(I D_{A}, I D_{B}, g^{b}, g^{a}\right), f_{s k}\left(I D_{A}, I D_{B}, g^{a}\right)$

7. $A \rightarrow B: f_{s k}\left(I D_{A}, I D_{B}, g^{b}\right)$

(3) Juang's three-party key agreement protocol [9]: The following protocol shows Juang's three-party password-based authenticated key agreement protocol. Juang's protocol requires totally 6 times symmetric encryption and 6 times symmetric decryption.

- Shared Information: $h(\cdot), p, q, g$.

- Information held by User $A$ : $I D_{A}, p w_{A}$, Token $\left(v_{A}=h\left(I D_{A}, x\right), h(\cdot), p, q, g\right)$.

- Information held by User $B: I D_{B}, p w_{B}$, Token $\left(v_{B}=h\left(I D_{B}, x\right), h(\cdot), p, q, g\right)$.

- Information held by Remote Server $S: x$.

1. $A \rightarrow B: r_{A}, I D_{A}, E_{v_{A}}\left(g^{a}, h\left(I D_{A}, I D_{B}, r_{A}\right)\right)$

2. $B \rightarrow S: r_{A}, r_{B}, I D_{A}, I D_{B}, E_{v_{A}}\left(g^{a}, h\left(I D_{A}, I D_{B}, r_{A}\right)\right)$, $E_{v_{B}}\left(g^{b}, h\left(I D_{A}, I D_{B}, r_{B}\right)\right)$

3. $S \rightarrow B: E_{v_{B}}\left(g^{a}, r_{A}, r_{B}+1\right), E_{v_{A}}\left(g^{b}, r_{B}, r_{A}+1\right)$

4. $B \rightarrow A: E_{s k}\left(r_{A}+1\right), E_{v_{A}}\left(g^{b}, r_{B}, r_{A}+1\right)$

5. $A \rightarrow B: E_{s k}\left(r_{B}+1\right)$ 


\section{Proposed Three-Party TAKE Protocol}

This section presents three-party token-based authenticated key establishment (3TAKE) protocol. 3TAKE protocol is composed of two protocols, which are key transport (3TAKT) and key agreement (3TAKA).

\subsection{Three-Party Key Transport}

This subsection proposes three-party token-based key transport (3TAKT) protocol. 3TAKT protocol is composed of two phases, which are the registration phase and the session key transport phase.

Registration Phase: User $U_{i}$ freely chooses his/her $I D_{i}$, password $p w_{i}$ and random nonce $n_{i}$, and interactively submits $\left\{I D_{i}, p w_{i} \oplus n_{i}\right\}$ to the remote server $S$. These private data must be sent in person or over a secure channel. $U_{i}$ also imprints his/her biometric impression at the sensor, and then $S$ performs the following operations:

R.1: Computes $v_{i}=h\left(I D_{i}, x\right), w_{i}=v_{i} \oplus h\left(p w_{i} \oplus n_{i}, S_{i}\right)$ and $x_{i}=h\left(v_{i}\right)$, where $S_{i}$ is the extracted biometric template of $U_{i}$.

R.2: Writes the secure information $\left\{w_{i}, x_{i}, S_{i}, h(\cdot)\right\}$ to the memory of $U_{i}$ 's token and issue the token to $U_{i}$ through a secure channel.

Upon receiving the token, $U_{i}$ enters $n_{i}$ into his/her token.

Key Transport Phase: Figure 1 shows the session key transport phase of 3TAKT protocol. The session key transport phase performs as follows:

In the session key transport phase, after getting the token from $S, A$ can use it when he/she securely communicates with $B$. If $A$ wants to negotiate a session key with $B$, he/she opens the login application software, enters $I D_{A}$ and $p w_{A}$, and imprints biometric at the sensor. For simplicity, we omit the verification process to check $h\left(v_{A}\right)$ with the stored $x_{A}$ from this phase. If $U_{A}$ is successfully verified by his/her biometric, $A$ 's token performs the following operations:

$\mathrm{S} .1 A \rightarrow S: I D_{A}, E_{v_{A}}\left(I D_{A}, I D_{B}, r_{A}\right)$

$A$ 's token computes $v_{A}=w_{A} \oplus h\left(p w_{A}, n_{A}\right)$, chooses a random nonce $r_{A}$, and sends his/her $I D_{A}$ and the encrypted message $E_{v_{A}}\left(I D_{A}, I D_{B}, r_{A}\right)$ to $S$.

$\mathrm{S} .2 S \rightarrow B: E_{v_{B}}\left(I D_{B}, I D_{A}, r_{A} \oplus s k, s k\right)$

Upon receiving the message in Step S.1, $S$ first computes $v_{A}=h\left(I D_{A}, x\right)$ using his/her master secret key $x$ and then decrypts the received message $E_{v_{A}}\left(I D_{A}, I D_{B}, r_{A}\right)$. Then, $S$ checks if the message contain the $A$ 's identity $I D_{A}$. If the identity is not valid, $S$ rejects this request. If $I D_{A}$ is valid, $S$ computes $v_{B}=h\left(I D_{B}, x\right)$ and generates the session key $s k$ for $A$ and $B$. Finally, $S$ sends the encrypted message $E_{v_{B}}\left(I D_{B}, I D_{A}, r_{A} \oplus s k, s k\right)$ to $B$. 
Shared Information: $h(\cdot)$.

Information held by User $A$ : $I D_{A}, p w_{A}$, Token $\left(w_{A}=v_{A} \oplus h\left(p w_{A}, n_{A}\right), n_{A}, h(\cdot)\right)$.

Information held by User $B: I D_{B}, p w_{B}$, Token $\left(w_{B}=v_{B} \oplus h\left(p w_{B}, n_{B}\right), n_{B}, h(\cdot)\right)$.

Information held by Remote Server $S: x$.

\section{User $A$}

Server $S$

User $B$

Insert $I D_{A}, p w_{A}$

$v_{A} \leftarrow w_{A} \oplus h\left(p w_{A}, n_{A}\right)$

Choose random $r_{A}$

$$
\begin{aligned}
& \frac{I D_{A}, E_{v_{A}}\left(I D_{A}, I D_{B}, r_{A}\right)}{v_{A} \leftarrow h\left(I D_{A}, x\right)} \\
& \text { Decrypt } E_{v_{A}}\left(I D_{A}, I D_{B}, r_{A}\right) \\
& \text { Abort if } I D_{A} \neq I D_{A} \\
& v_{B} \leftarrow h\left(I D_{B}, x\right) \\
& \text { Choose } s k \\
& E_{v_{B}}\left(I D_{B}, I D_{A}, r_{A} \oplus s k, s k\right)
\end{aligned}
$$

Insert $I D_{B}, p w_{B}$ $v_{B} \leftarrow w_{B} \oplus h\left(p w_{B}, n_{B}\right)$ Decrypt $E_{v_{B}}\left(I D_{B}, I D_{A}, r_{A} \oplus s k, s k\right)$

$r_{A} \oplus s k, h\left(I D_{B}, I D_{A}, s k\right)$

Abort if $I D_{B} \neq I D_{B}$

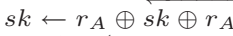

Verify $h\left(I D_{B}, I D_{A}, s k\right)$

$$
\text { Verify } h\left(\overrightarrow{I D}_{A}, I D_{B}, s k\right)
$$

Shared Session Key between $A$ and $B: s k$

Fig. 1. 3TAKT protocol

$\mathrm{S} .3 B \rightarrow A: r_{A} \oplus s k, h\left(I D_{B}, I D_{A}, s k\right)$

Upon receiving the message in Step S.2, $B$ inputs his/her identity $I D_{B}$ and password $p w_{B}$ to his/her token. Then, $B$ 's token computes $v_{B}=w_{B} \oplus$ $h\left(p w_{B}, n_{B}\right)$ and decrypts the received message $E_{v_{B}}\left(I D_{B}, I D_{A}, r_{A} \oplus s k, s k\right)$. Then, $B$ checks if the message contain his/her identity $I D_{B}$. If the identity is not valid, $B$ rejects this request. If $I D_{B}$ is valid, $B$ computes $h\left(I D_{B}, I D_{A}, s k\right)$ and sends it with $r_{A} \oplus$ sk to $A$.

$\mathrm{S} .4 A \rightarrow B: h\left(I D_{A}, I D_{B}, s k\right)$

After receiving the message in Step S.3, $A$ extracts shared session key $s k$ by computing $r_{A} \oplus s k \oplus r_{A}$ and checks if the hash value $h\left(I D_{B}, I D_{A}, s k\right)$ is correct. If yes, $A$ computes and sends $h\left(I D_{A}, I D_{B}, s k\right)$ back to $B$.

S.5 After receiving the message in Step S.4, $B$ checks if the hash value $h\left(I D_{A}\right.$, $\left.I D_{B}, s k\right)$ is correct. Then $A$ and $B$ can use the shared secret session key $s k$ in private communication soon.

\subsection{Three-Party Key Agreement}

This subsection proposes three-party token-based key agreement (3TAKA) protocol. 3TAKA protocol is composed of two phases, which are the registration phase and the session key transport phase. 
Registration Phase: The registration phase is same as 3TAKT.

Key Agreement Phase: Figure 2 shows the session key agreement phase of 3TAKT protocol. The session key agreement phase performs as follows:

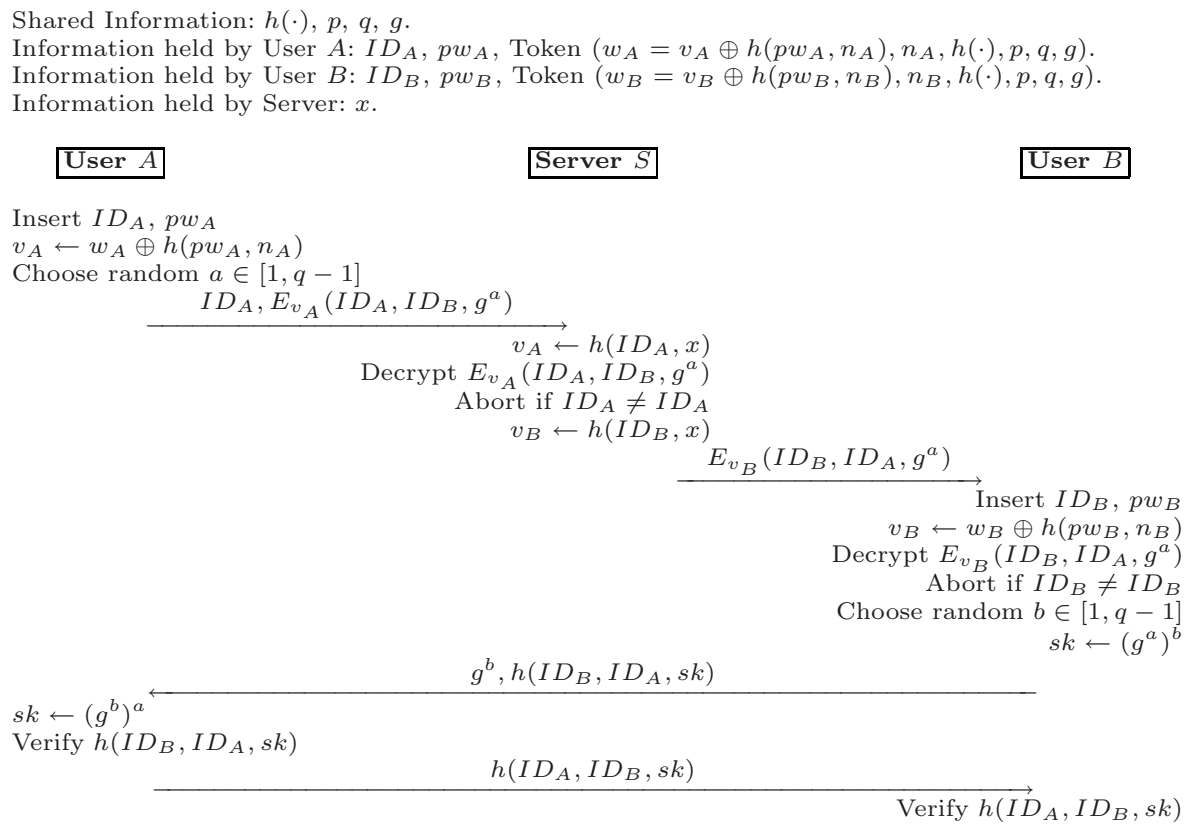

Insert $I D_{A}, p w_{A}$

$v_{A} \leftarrow w_{A} \oplus h\left(p w_{A}, n_{A}\right)$

Choose random $a \in[1, q-1]$

$\stackrel{I D_{A}, E_{v_{A}}\left(I D_{A}, I D_{B}, g^{a}\right)}{v_{A} \leftarrow h\left(I D_{A}, x\right)}$

Decrypt $E_{v_{A}}\left(I D_{A}, I D_{B}, g^{a}\right)$

Abort if $I D_{A} \neq I D_{A}$

$v_{B} \leftarrow h\left(I D_{B}, x\right)$

$\stackrel{E_{v_{B}}\left(I D_{B}, I D_{A}, g^{a}\right)}{\longrightarrow}$ Insert $I D_{B}, p w_{B}$

$v_{B} \leftarrow w_{B} \oplus h\left(p w_{B}, n_{B}\right)$

Decrypt $E_{v_{B}}\left(I D_{B}, I D_{A}, g^{a}\right)$

Abort if $I D_{B} \neq I D_{B}$

Choose random $b \in[1, q-1]$ $s k \leftarrow\left(g^{a}\right)^{b}$

$g^{b}, h\left(I D_{B}, I D_{A}, s k\right)$

$s k \leftarrow\left(g^{b}\right)^{a}$

Verify $h\left(I D_{B}, I D_{A}, s k\right)$

Verify $h\left(\overrightarrow{I D}_{A}, I D_{B}, s k\right)$

Shared Session Key between $A$ and $B: s k=g^{a b}$

Fig. 2. 3TAKA protocol

In the session key agreement phase, after getting the token from $S, A$ can use it when he/she securely communicates with $B$. If $A$ wants to negotiate a session key with $B$, he/she opens the login application software, enters $I D_{A}$ and $p w_{A}$. Then $A$ 's token performs the following operations. For simplicity, we omit $(\bmod p)$ from expressions.

\section{$\mathrm{S} .1 A \rightarrow S: I D_{A}, E_{v_{A}}\left(I D_{A}, I D_{B}, g^{a}\right)$}

User $A$ 's token computes $v_{A}=w_{A} \oplus p w_{A}$, chooses a random number $a \in$ $[1, q-1]$, and computes $g^{a}$. Then, $A$ sends his/her $I D_{A}$ and the encrypted message $E_{v_{A}}\left(I D_{A}, I D_{B}, g^{a}\right)$ to $S$.

$\mathrm{S} .2 S \rightarrow B: E_{v_{B}}\left(I D_{B}, I D_{A}, g^{b}\right)$

Upon receiving the message in S.1, $S$ first computes $v_{A}=h\left(I D_{A}, x\right)$ using his/her master secret key $x$ and then decrypts the received message $E_{v_{A}}\left(I D_{A}, I D_{B}, g^{a}\right)$. Then, $S$ checks if the message contain the $A$ 's identity 
$I D_{A}$. If the identity is not valid, $S$ rejects this request. If $I D_{A}$ is valid, $S$ computes $v_{B}=h\left(I D_{B}, x\right)$ and sends the encrypted message $E_{v_{B}}\left(I D_{B}, I D_{A}, g^{a}\right)$ to $B$.

$\mathrm{S} .3 B \rightarrow A: g^{b}, h\left(I D_{B}, I D_{A}, g^{a}, s k\right)$

Upon receiving the message in S.2, $B$ inputs his/her identity $I D_{B}$ and password $p w_{B}$ to his/her token. Then, $B$ 's token computes $v_{B}=w_{B} \oplus$ $h\left(p w_{B}, n_{B}\right)$ and decrypts the received message $E_{v_{B}}\left(I D_{B}, I D_{A}, g^{b}\right)$. Then, $B$ checks if the message contain his identity $I D_{B}$. If the identity is not valid, $B$ rejects this request. If $I D_{B}$ is valid, $B$ chooses a random number $b \in[1, q-1]$, and computes $g^{b}$ and the shared session key $s k=\left(g^{a}\right)^{b}=g^{a b}$. Finally, $B$ sends $g^{b}$ and $h\left(I D_{B}, I D_{A}, g^{a}, s k\right)$ to $A$.

$\mathrm{S} .4 A \rightarrow B: h\left(I D_{A}, I D_{B}, g^{b}, s k\right)$

After receiving the message in $\mathrm{S} .3, A$ computes the shared session key $s k=$ $\left(g^{b}\right)^{a}=g^{a b}$ and checks if the hash value $h\left(I D_{B}, I D_{A}, g^{a}, s k\right)$ is correct. If yes, $A$ computes and sends $h\left(I D_{A}, I D_{B}, g^{b}, s k\right)$ back to $B$.

S.5 After receiving the message in $\mathrm{S} .4, B$ checks if the hash value $h\left(I D_{A}, I D_{B}\right.$, $\left.g^{b}, s k\right)$ is correct. Then $A$ and $B$ can use the shared secret session key $s k=$ $g^{a b}$ in private communication soon.

\section{Security Analysis}

This section provides the proof of correctness of the proposed 3TAKE. Here, four security properties 18193: guessing attacks, replay attacks, mutual authentication and perfect forward secrecy, would be considered for 3TAKE. Under the definitions of Section 2, the following theorems are used to analyze the three security properties in $3 \mathrm{TAKE}$ protocols.

Theorem 1. The proposed $3 T A K E$ protocol can resist guessing attacks.

Proof. The undetectable on-line password guessing attack will fail, since after Step S.2 of 3TAKE, $S$ can authenticate $A$. The off-line password guessing attack will not work against $3 \mathrm{TAKT}$ since the password $p w_{A}$ is only used for protecting the corresponding token, and no verifiable information is encrypted by passwords. Also, the secret $w_{A}=v_{A} \oplus h\left(p w_{A}, n_{A}\right)$ is stored in A's token. Only the legal user $A$ has his/her password $p w_{A}$ can compute the secret $v_{A}=w_{A} \oplus h\left(p w_{A}, n_{A}\right)$ and then use his/her token.

Therefore, the proposed 3TAKE protocol can resist guessing attacks.

Theorem 2. The proposed $3 T A K E$ protocol can resist replay attacks.

Proof. In the proposed 3TAKT, the replay attacks fail because the freshness of the messages transmitted in the session key transport phase is provided by the random nonce $r_{A}$ and session key $s k$. Only $A$ and $B$, who can get the session key $s k$, can embed the session key $s k$ in the hashed messages $h\left(I D_{B}, I D_{A}, r_{A}, s k\right)$ generated by $A$ of Step S.4 and $h\left(I D_{A}, I D_{B}, s k\right)$ generated by $B$ of Step S.5, respectively. 
In the proposed 3TAKA, the replay attacks fail because the freshness of the messages transmitted in the session key agreement phase is provided by the exponents $a$ and $b$. Except for $A$, only $B$ who can compute the session key $s k$ can embed the nonce $g^{a}$ and the session key $s k$ generated by $A$ in the hashed message $h\left(I D_{B}, I D_{A}, g^{a}, s k\right)$ of Step S.4. Except for $B$, only $A$ who can compute the session key $s k$ can embed the nonce $g^{b}$ and the session key $s k$ generated by $B$ in the hashed message $h\left(I D_{A}, I D_{B}, g^{b}, s k\right)$ of Step S.5.

Therefore, the proposed 3 TAKE protocol can resist replay attacks.

Theorem 3. The proposed 3TAKE protocol provides the mutual authentication.

Proof. In 3TAKT protocol, the goal of mutual authentication is to generate an agreed session key $s k$ between $A$ and $B$ for $i$-th session. In Step S.3 of the session key transport phase, after $B$ receiving the encrypted message $E_{v_{B}}\left(I D_{B}, I D_{A}, r_{A}\right)$ from $S$, he/she will check if the encrypted message contains the identity $I D_{B}$. Since the identity $I D_{B}$ is encrypted by the secret key $v_{B}$ shared between $S$ and $B, B$ will believe the $i$-th random value $r_{A}$ was originally sent from $A$, verified by the trusted server $S$ in Step S.2 and then sent to him/her. $B$ then can compute the session key $s k$. In Step S.4, after $A$ receiving the encrypted message $r_{B}$ and $h\left(I D_{B}, I D_{A}, r_{A}, s k\right)$ from $B$, he/she will check if the encrypted message contains the random value $r_{A}$ and the session key $s k$. Since the hashed message included $r_{A}$ and $s k, A$ will believe $h\left(I D_{B}, I D_{A}, r_{A}, s k\right)$ was originally sent from $B$, verified by the trusted server $S$ in Step S.2 and then sent to him/her.

In 3TAKA protocol, the goal of mutual authentication is to generate an agreed session key $s k$ between $A$ and $B$ for $i$-th session. In Step S.3 of the session key agreement phase, after $B$ receiving the encrypted message $E_{v_{B}}\left(I D_{B}, I D_{A}, g^{a}\right)$ from $S$, he will check if the encrypted message contains the identity $I D_{B}$. Since the identity $I D_{B}$ is encrypted by the secret key $v_{B}$ shared between $S$ and $B$, $B$ will believe the $i$-th random value $g^{a}$ was originally sent from $A$, verified by the trusted server $S$ in Step S.2 and then sent to him/her. $B$ then can compute the session key $s k$. In Step S.4, after $A$ receiving the encrypted message $g^{b}$ and $h\left(I D_{B}, I D_{A}, g^{a}, s k\right)$ from $B$, he/she will check if the encrypted message contains the random value $g^{a}$ and the session key $s k$. Since the hashed message included the shared session key $s k$ between $A$ and $B, A$ will believe the $i$-th random value $g^{b}$ was originally sent from $B$, verified by the trusted server $S$ in Step S.2 and then sent to him/her. $A$ then can compute the session key $s k$.

Therefore, the proposed 3TAKE protocol provides the mutual authentication.

Theorem 4. The proposed 3TAKA protocol provides perfect forward secrecy.

Proof. A disclosed long-lived secret key $v_{A}, v_{B}$ or $x$ cannot derive the session key $s k=g^{a b}$ used before because without getting the used random exponents $a$ and $b$, nobody can compute the used session key $s k$. If an attacker wiretaps all conversations of the medium and derives some used random values $g^{a}$ and $g^{b}$, he/she could not compute the used session key $s k$. This problem is the DiffieHellman key exchange algorithm.

Therefore, the proposed 3TAKA protocol provides perfect forward secrecy. 


\section{$5 \quad$ Efficiency Analysis}

This section discusses the efficiency of newly proposed 3TAKT and 3TAKA protocols.

\subsection{Efficiency of 3TAKT Protocol}

In recent years, a variety of protocols, which belong to three-part authenticated key transport (3AKT) protocol, have been proposed. This subsection compares some well-known traditional 3AKT protocols including the optimal GLNS protocol [7, the improved K1P protocol [14, the extension of Otway-Rees protocol [3], and KTAP-1 of Yeh [15], with our newly proposed 3TAKT protocol. Optimal GLNS protocol improved the performance of their original GLNS protocol 7 ] and improved K1P [14] improved three-way K1P [16] which is vulnerable to a straight replay attacks.

We focus on several items such as the number of rounds, the number of random numbers, the number of symmetric encryption/decryption operations, and the number of asymmetric encryption/decryption operations. We ignore other comparisons like the total amount of data transferred because these items are varying for different security levels. Table 1 shows the comparison results.

In Table1, we can find that proposed 3TAKT is the most efficient in number of rounds, number of random numbers, and other computational costs. That is, in 3TAKT, the number of symmetric key encryption/decryption operations among three parties is reduced by about $34 \sim 67 \%$ compared with the related protocols. Furthermore, the number of rounds is smaller one round than the related protocols and the asymmetric key encryption/decryption operations do not need to transport a session key and authenticate between two users and a server.

Table 1. Comparison of efficiency with related 3AKT protocols

\begin{tabular}{|l||c|c|c|c|c|}
\hline \hline Computations costs & $\begin{array}{c}\text { Optimal } \\
\text { GLNS } \\
(1995)\end{array}$ & $\begin{array}{c}\text { Extension of } \\
\text { OR } \\
(1997)\end{array}$ & $\begin{array}{c}\text { Improved } \\
\text { K1P } \\
(1999)\end{array}$ & $\begin{array}{c}\text { KTAP-1 } \\
\text { (2003) }\end{array}$ & 3TAKT \\
\hline \hline $\begin{array}{l}\text { \# of asymmetric } \\
\text { enc/decryption }\end{array}$ & $2 / 2$ & 0 & $2 / 2$ & $2 / 2$ & 0 \\
\hline $\begin{array}{l}\text { \# of symmetric } \\
\text { enc/decryption }\end{array}$ & $4 / 4$ & $6 / 6$ & $4 / 4$ & $3 / 3$ & $2 / 2$ \\
\hline \# of random numbers & 10 & 3 & 5 & 4 & 3 \\
\hline \# of rounds & 5 & 5 & 5 & 5 & 4 \\
\hline \hline
\end{tabular}

\subsection{Efficiency of 3TAKA Protocol}

In recent years, a variety of protocols, which belong to three-part authenticated key agreement (3AKA) protocol, have been proposed. This subsection compares some well-known traditional 3AKA protocols including STW 13, LSH [1], 
LSSH [12], ECC [17], KAAP-1 [15], and Juang [9], with our newly proposed 3TAKA protocol. Table 2 shows the comparison results.

In Table 2, we can find that proposed 3TAKA protocol is the most efficient in number of rounds, number of random numbers, and other computational costs. That is, in 3TAKA, the number of exponentiations among three parties is same or reduced by about $34 \sim 60 \%$ and the number of symmetric key encryption/decryption operations among three parties is reduced by about $34 \sim$ $67 \%$ compared with the related protocols, respectively. Furthermore, the number of rounds is smaller one round than the related protocols and the asymmetric key encryption/decryption operations do not need to establish a session key and authenticate between two users and a server.

Table 2. Comparison of efficiency with related 3AKA protocols

\begin{tabular}{|l||c|c|c|c|c|c|c|}
\hline \hline Computations costs & $\begin{array}{c}\text { STW } \\
(1995)\end{array}$ & $\begin{array}{c}\text { LSH } \\
(2000)\end{array}$ & $\begin{array}{c}\text { LSSH } \\
(2001)\end{array}$ & $\begin{array}{c}\text { KAAP-1 } \\
(2003)\end{array}$ & $\begin{array}{c}\text { ECC } \\
(2004)\end{array}$ & $\begin{array}{c}\text { Juang } \\
(2004)\end{array}$ & 3TAKA \\
\hline \hline $\begin{array}{l}\text { \# of asymmetric } \\
\text { enc/decryption }\end{array}$ & 0 & $2 / 2$ & 0 & $2 / 2$ & 0 & 0 & 0 \\
\hline $\begin{array}{l}\text { \# of symmetric } \\
\text { enc/decryption }\end{array}$ & 0 & $3 / 3$ & $2 / 2$ & $3 / 3$ & $2 / 2$ & $6 / 6$ & $2 / 2$ \\
\hline \# of exponentials & 6 & 8 & 10 & 4 & 10 & 4 & 4 \\
\hline \# of hash functions & 0 & 2 & 10 & 0 & 16 & 8 & 4 \\
\hline \# of random numbers & 3 & 5 & 4 & 3 & 5 & 4 & 2 \\
\hline \# of rounds & 5 & 5 & 5 & 5 & 5 & 5 & 4 \\
\hline \hline
\end{tabular}

\section{Conclusions}

This paper proposed new efficient and secure three-party token-based authenticated key establishment (3TAKE) protocol based on symmetric key cryptosystem to minimize the complexity of symmetric key encryption/decryption operation among all users and fit three-party communication. In 3TAKE, the number of exponentiations among three parties is same or reduced by about $34 \sim 60 \%$ and the number of symmetric key encryption/decryption operations among three parties is reduced by about $34 \sim 67 \%$ compared with the related protocols, respectively. Furthermore, the number of rounds is smaller one round than the related protocols and the asymmetric key encryption/decryption operations do not need to establish a session key and authenticate between two users and a server.

\section{Acknowledgements}

We would like to thank the anonymous reviewers for their helpful comments in improving our manuscript. This research was supported by the MIC of Korea, under the ITRC support program supervised by the IITA (IITA-2006-C10900603-0026). 


\section{References}

1. Shneier, B.: Applied Cryptography, 2nd edn. John Wiley \& Sons, Inc., Chichester (1996)

2. Boyd, C., Mathuria, A.: Protocols for Authentication and Key Establishment. Springer, Heidelberg (2003)

3. Menezes, A.J., Oorschot, P.C., Vanstone, S.A.: Handbook of Applied Cryptograph. CRC Press, New York (1997)

4. Mao, W.: Modern Cryptography Theory \& Practice. Prentice Hall, Englewood Cliffs (2004)

5. Stinson, D.: Cryptography Theory and Practice, 2nd edn. Chapman \& Hall/CRC (2002)

6. Blake-Wilson, S., Menezes, A.: Authenticated Diffie-Hellman Key Agreement Protocols. In: Tavares, S., Meijer, H. (eds.) SAC 1998. LNCS, vol. 1556, pp. 339-361. Springer, Heidelberg (1999)

7. Gong, L., Lomas, M., Needham, R., Saltzer, J.: Protecting Poorly Chosen Secrets from Guessing Attacks. IEEE Journal on Selected Areas in Communications 11(5), 648-656 (1993)

8. Gong, L.: Optimal Authentication Protocols Resistant to Password Guessing Attacks. In: Proc. of the 8th IEEE Computer Security Foundation Workshop, pp. 24-29 (1995)

9. Juang, W.S.: Efficient Three-Party Key Exchange using Smart Cards. IEEE Transactions on Consumer Electronics 50(2), 619-624 (2004)

10. Keung, S., Siu, K.: Efficient Protocols Secure Against Guessing and Replay Attacks. In: Proc. of the Fourth International Conference on Computer Communications and Networks, pp. 105-112 (1995)

11. Lin, C., Sun, H., Hwang, T.: Three-party Encrypted Key Exchange: Attacks and a Solution. ACM Operating Systems Review 34(4), 12-20 (2000)

12. Lin, C., Sun, H., Steiner, M., Hwang, T.: Three-party Encrypted Key Exchange Without Server Public-Keys. IEEE Communication Letters 5(12), 497-499 (2001)

13. Steiner, M., Tsudik, G., Waidner, M.: Refinement and Extension of Encrypted Key Exchange. ACM Operating Systems Review 29(3), 22-30 (1995)

14. Kwon, T., Kang, M., Jung, S., Song, J.: An Improvement of the Password based Authentication Protocol (K1P) on Security against Replay Attacks. IEICE Transactions on Communications E82-B(7), 991-997 (1999)

15. Yeh, H.T.: User Authentication and Key Exchange Protocols Suitable for Diverse Circumstances, Ph.D. Thesis, Southern Taiwan University of Technology, Taiwan, pp. 1-82 (2003)

16. Kwon, T., Kang, M., Song, J.: An Adaptable and Reliable Authentication Protocol for Communication Networks. In: Proc. of IEEE INFOCOM 1997, pp. 737-744 (1997)

17. Chang, C., Chang, Y.: A Novel Three-party Encrypted Key Exchange Protocol. Computer Standards \& Interfaces 26(5), 471-476 (2004)

18. Lin, C.L., Hwang, T.: A Password Authentication Scheme with Secure Password Updating. Computers \& Security 22(1), 68-72 (2003)

19. Liao, I.E., Lee, C.C., Hwang, M.S.: A Password Authentication Scheme over Insecure Networks. Journal of Computer and System Sciences 72(4), 727-740 (2006) 